\title{
Iron-fortified recombinant Saccharomyces cerevisiae producing Sus scrofa ferritin heavy-chain recovers iron deficiency in mice
}

\author{
Hwan Lim ${ }^{1,2}$, Jong-Taek Kim, Myoung-Dong Kim ${ }^{3}$, Ki-Jong Rhee ${ }^{4}$, Bae Dong Jung ${ }^{1, *}$ \\ ${ }^{1}$ College of Veterinary Medicine, Kangwon National University, Chuncheon 200-701, Korea \\ ${ }^{2}$ Adbiotech, Chuncheon 200-883, Korea \\ ${ }^{3}$ Department of Food Science and Biotechnology, Kangwon National University, Chuncheon 200-701, Korea \\ ${ }^{4}$ Department of Biomedical Laboratory Science, College of Health Sciences, Yonsei University, Wonju 220-710, Korea
}

(Received: July 4, 2012; Revised: November 12, 2012; Accepted: November 16, 2012)

\begin{abstract}
In this study, we produced iron-fortified yeast (Saccharomyces cerevisiae) producing Sus scrofa ferritin heavychain to provide iron supplementation in anemic piglets. We determined whether iron-ferritin accumulated in recombinant yeasts could improve iron deficiency in mice. C57BL/6 male mice exposed to Fe-deficient diet for 2 weeks were given a single dose of ferrous ammonium sulfate (FAS), ferritin-producing recombinant yeast (APO), or APO reacted with iron $\left(\mathrm{Fe}^{2+}\right)$ (FER). The bioavailability of recombinant yeasts was examined by measuring body weight gain, hemoglobin concentration and hematocrit value 1 week later. In addition, ferritin protein levels were evaluated by western blot analysis and iron stores in tissues were measured by inductively coupled plasma spectrometer. We found that anemic mice treated with FER exhibited increased levels of ferritin heavy-chain in spleen and liver. Consistently, this treatment restored the iron concentration in these tissues. In addition, this treatment significantly increased hemoglobin value and the hematocrit ratio. Furthermore, FER treatment significantly enhanced body weight gain. These results suggest that the iron-fortified recombinant yeast strain is bioavailable.
\end{abstract}

Keywords : anemia, ferritin, iron, Saccharomyces cerevisiae, Sus scrofa

\section{Introduction}

Iron deficiency is one of the most common causes of anemia in humans [4, 8]. Iron deficiency anemia is commonly observed during the neonatal phase and early childhood [5]. Iron deficiency anemia is also recognized as a serious disorder in many livestock especially suckling piglets. One main reason for iron deficiency in newborn piglets is the rapid increase in blood volume and subsequent increase in the red blood cell numbers during the rapid growth phase of these animals $[13,18]$. As a consequence, the majority of the iron present in plasma is incorporated into hemoglobin of erythroid precursors in the bone marrow. Since sow's milk does not contain sufficient amount of iron to maintain optimal levels of hemoglobin in suckling piglets, iron supplements must be provided to maintain health [2]. The current practice in the pig industry is administering intramuscular injections of large amounts of iron dextran $[6,18]$. However, this regimen can cause muscular necrosis and iron dextran can be rapidly metabolized in muscle [13]. Iron deficiency can also be prevented by providing oral iron supplements such as ferrous sulfate. However, iron overdose can lead to gastrointestinal malabsorption. Moreover, inappropriately high intake of supplemental iron in the diet may perturb the tight control of systemic iron metabolic processes $[1,8,13]$.

The major intracellular iron-storage protein is ferritin. Ferritin acts as a supplier of iron and as a reserve thereby providing iron to cells of developing animals [10, 12, 19]. Studies in rats have demonstrated that a dietary supplement of purified ferritin and soybean meal could recover iron deficiency [1]. In pigs, ferritin concentration in the serum and liver rapidly decrease after birth [7, 14].

The food yeast, Saccharomyces $(S$.) cerevisiae, is an organism generally regarded as safe and has been used as a host for expression of a variety of heterologous genes. Consequently, $S$. cerevisiae has been used in livestock feeds and as food supplements in humans [3]. In this study, we constructed iron-fortified recombinant $S$. cerevisiae by expressing Sus scrofa ferritin heavy-chain genes in S. cerevisiae for a modified strategy for iron supplementation of anemic piglets. As a preliminary step, we determined whether iron-ferritin accumulated in recombinant yeasts could improve iron deficiency in mice.

*Corresponding author

Tel: +82-33-250-8674, Fax: +82-33-244-2367

E-mail: bdjung@kangwon.ac.kr 


\section{Materials and Methods}

\section{Construction of the expression vector}

Sus scrofa ferritin heavy-chain cDNAs were amplified by reverse transcription polymerase chain reaction (RT-PCR) using total RNA from pig spleen. The primers for cloning were based on the Sus scrofa ferritin heavy-chain sequences from GeneBank (Accession No. D15071) were commercially synthesized (Bioneer, Korea). The primer sequences were as follows: F-EcoR1: 5'-aattgaattcatgacgacctcgtgctcc-3'; R-Xho1: 5'-aattctcgagttagctctcactgctcccca-3'. The PCR products were cloned into pGEM-T (Promega, USA). LB medium $(0.5 \%$ bacto-yeast extract, $1 \%$ bacto-tryptone peptone, and $1 \%$ $\mathrm{NaCl}$ ) supplemented with $100 \mu \mathrm{g} / \mathrm{mL}$ ampicillin was used for plasmid DNA preparation. Plasmid DNA was isolated using the AccuPrep plasmid Mini Extraction Kit (Bioneer). All DNA sequences were determined at Macrogen (Korea). A multicopy plamid, p426GPD $(7.28 \mathrm{~kb})$, containing the $S$. cerevisiae GPD promoter and $\mathrm{CYC1}$ terminator was used for expression of Sus scrofa H-ferritin.

Yeast strains, transformation, and culture conditions

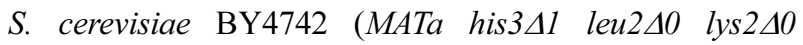
ura3 $\Delta 0$ ) was used as a host strain for the heterologous expression of the Sus scrofa ferritin heavy-chain genes. S. cerevisiae BY4742 was transformed by the lithium acetate method [9]. Transformed yeasts were cultured in YEPD (1\% bacto-yeast extract, $2 \%$ bacto-proteose peptone, and $2 \%$ glucose) media. S. cerevisiae cells carrying plasmid were grown in synthetic complete medium lacking uracil and containing $2 \%$ galactose instead of glucose for the expression of the Sus scrofa ferritin heavy-chain. The $S$. cerevisiae transformants were grown at $30^{\circ} \mathrm{C}$ for $12 \mathrm{~h}$ with continuous agitation (200 $\mathrm{rpm}$ ) and harvested by centrifugation at $5,000 \mathrm{~g}$ for $10 \mathrm{~min}$ $\left(4^{\circ} \mathrm{C}\right)$. Cells were washed twice with $20 \mathrm{mM}$ of 3 -(N-morholino) propane sulfonic acid buffer ( $\mathrm{pH}$ 6.5). After preincubation cells for $30 \mathrm{~min}$ in this buffer, which also contained $5 \%(\mathrm{w} / \mathrm{v})$ glucose, yeast cells reacted by $14.3 \mathrm{mM}$ of $\mathrm{Fe}$ $\left(\mathrm{NH}_{4}\right)_{2}\left(\mathrm{SO}_{4}\right)_{2}$ at $30^{\circ} \mathrm{C}$ for $2 \mathrm{~h}$ with continuous agitation $(200$ rpm) [3, 17]. Study by Seo et al. [16] showed that using this method, cellular iron incorporates at a concentration of 16.7 $\mathrm{mol} / \mathrm{g}$ of wet cell weight. We used this method to incorporate cellular iron into Sus scrofa ferritin heavy-chain expressing yeast. S. cerevisiae cells expressing Sus scrofa ferritin heavy-chain genes were denoted as ferritin-producing recombinant yeast (APO). S. cerevisiae cells expressing ferritin heavy-chain containing incorporated iron were denoted as APO reacted with iron $\left(\mathrm{Fe}^{2+}\right)$ (FER). Cell pellets of APO (3 g of wet weight) and FER ( $3 \mathrm{~g}$ of wet weight) were used for animal dietary treatment.

\section{Animals and diet supplement treatment}

All animals were treated in strict accordance with the NIH Guide for the Humane Care and Use of Laboratory Animals. Male C57BL/6J mice (Orient Bio, Korea) weighing $19 \pm 1 \mathrm{~g}$ were maintained on a $12 \mathrm{~h}$ light: $12 \mathrm{~h}$ dark cycle. The control group was fed an AIN-76A diet $(48 \mathrm{mg} \mathrm{Fe} / \mathrm{kg}$ diet; Dyets, USA). Iron deficient animals group were prepared by feeding them an AIN Fe-deficient diet ( $3 \mathrm{mg} \mathrm{Fe} / \mathrm{kg}$ diet) for 21 day (d). The four test groups were maintained under Fedeficient conditions. Fourteen days after the Fe-deficient diet, mice received a single dose of treatment plus ferrous ammonium sulfate (FAS; $100 \mathrm{mg} / \mathrm{kg}$, p.o., once in $14 \mathrm{~d}$ ), apoferritin $(3 \mathrm{~g} / \mathrm{kg}$, p.o., once in $14 \mathrm{~d})$ or FER (3 g/kg, p.o., once in $14 \mathrm{~d})$. Body weight of mice was measured every day throughout the duration of the experiment. Blood was collected by cardiac puncture at $21 \mathrm{~d}$. Hemoglobin levels were measured using a hemoglobin diagnostics kit (Yd-diagnostics, Korea) and hematocrit ratio was measured after blood centrifugation. The spleen and liver were harvested, weighed and used to determine iron concentration or for western blot analysis. The iron concentration was determined by inductively coupled plasma spectrometer (OPTIMA 7300 DV; Perkin-Elmer, USA).

\section{Western blot analysis}

Western blotting analysis was performed as described previously [11]. Tissues were homogenized in lysis buffer, containing $200 \mathrm{mM}$ Tris $\mathrm{HCl}$ (pH 6.8), 10\% SDS, 5 mM EGTA, $5 \mathrm{mM}$ EDTA, $10 \%$ glycerol and protease inhibitor cocktail (Sigma, USA). Cells lysates were centrifuged at 13,000 g for $30 \mathrm{~min}$ and the supernatant fraction was used for western blot analysis. Proteins $(25 \sim 30 \mu \mathrm{g} /$ lane $)$ were separated by $8 \%$ or $10 \%$ SDS-PAGE and the resolved proteins were transferred onto the PVDF membranes. Blocking was performed using PBS containing 3\% skim milk for $30 \mathrm{~min}$. Each blot was incubated overnight at $4^{\circ} \mathrm{C}$ with the primary antibody against $\beta$-actin $(1: 50,000$; Sigma), actin (1:1,000; Thermo, USA) or H-ferritin ( $1: 500$; abcam, UK). After washing in PBS, membranes were incubated with HRP-conjugated secondary anti-rabbit IgG (1:5,000; GE Healthcare, USA), antimouse $\operatorname{IgG}(1: 5,000$; Sigma) or anti-goat $\operatorname{IgG}(1: 5,000$; Sigma) for $2 \mathrm{~h}$. Subsequent visualization was performed using enhanced chemiluminescence system (ECL plus; GE Healthcare). Relative band intensities were quantified by PhotoCapt MW (ver. 10.01 for Windows; Vilber Lourmat, France).

\section{Statistical analysis}

Analysis of variance was performed with post hoc Tukey's test to determine whether dietary groups were significantly different from each other. Differences with a $p<0.05$ were considered significant. Statistical analysis was carried out using SPSS (SPSS, USA).

\section{Results}

Yeast cells containing either the empty expression vector or the expression vector containing the Sus scrofa ferritin heavy-chain cDNA were cultured for $12 \mathrm{~h}$ in $2 \%$ galactose medium. The crude cell extracts were used for the western blot analysis. The extracts from cells containing the empty 


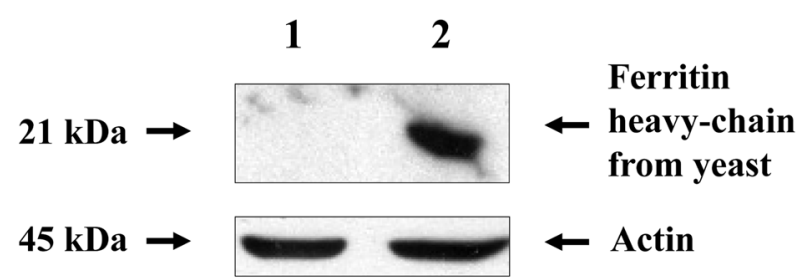

Fig. 1. Expression of Sus scrofa ferritin heavy-chain induced by galactose. Cultured cell was extracted by vortexing in the presence of glass beads and heated at $100^{\circ} \mathrm{C}$ for $3 \mathrm{~min}$. The lanes are: 1, protein from cells transformed with control empty expression vectors; 2, protein from cells transformed with Sus scrofa ferritin heavy-chain expression vectors. Proteins $(25 \mu \mathrm{g} /$ lane) were used for sodium dodecyl sulfate-polyacrylamide gel electrophoresis.

expression vector did not exhibit a band for the Sus scrofa ferritin heavy-chain $(21 \mathrm{kDa})$ (Fig. 1). On the other hand, cells containing the expression vector for Sus scrofa ferritin heavy-chain exhibited a band for the recombinant ferritin heavy-chain.

Body weight gain in mice given the different dietary formulations is shown in Table 1. Compared to the control mice, the lack of Fe supplement (Fe-def) resulted in a significant decrease in body weight gain ( $p<0.05 v s$. control). Mice administered either FAS or Sus scrofa ferritin heavychain expressing yeast (APO) did not gain body weight compared to the Fe-def mice. However, mice given iron accumulated yeast cells producing Sus scrofa ferritin heavy-chain (FER) exhibited significantly increased body weight gain $(p<0.05$ vs. Fe-def).

Alterations in hemoglobin and hematocrit levels are shown in Table 2. As expected, significant reduction in hemoglobin concentration and hematocrit ratios were observed in Fe-deficient group animals $(p<0.05 v s$. control). Treatment with FER significantly increased hemoglobin concentration and hematocrit ratio $(p<0.05$ vs. Fe-def). Interestingly, treatment with APO also enhanced hemoglobin value and hematocrit ratio ( $p<0.05 v s$. Fe-def). Treatment with FAS significantly induced hemoglobin value $(p<0.05 v s$. Fe-def) but did not affect hematocrit ratio.

To examine whether administration of recombinant yeast producing Sus scrofa ferritin heavy-chain affects organ iron stores, iron concentration was measured in the spleen and liver (Table 3). In mice fed with Fe-deficient diet, iron concentration in spleen and liver significantly decreased compared to the control diet group. Treatment with APO significantly increased iron concentration in the spleen and in the liver $(p<0.05 v s$. Fe-def). Mice treated with FER had higher concentration of iron in the spleen compared to Fe-def mice and APO mice $(p<0.05)$. However, there was no significant increase in iron concentration in the liver of FER mice compare to APO mice. Interestingly, treatment with FAS significantly increased the iron concentration in spleen $(p<0.05 v s$. Fe-def) but not in the liver.

We examined protein levels of ferritin heavy-chain in spleen and liver in the different groups of mice (Fig. 2). As expected, ferritin heavy-chain levels were significantly decreased in absence of iron in the diet of both spleen $(p<$ $0.01 v s$. control) and liver $(p<0.05 v s$. control). Mice admin-

Table 1. Changes in body weight of iron deficiency mice

\begin{tabular}{ccccc}
\hline \hline & $\begin{array}{c}\text { Body weight } \\
(\mathrm{g}, 14 \mathrm{~d})\end{array}$ & $\begin{array}{c}\text { Body weight gain } \\
(\mathrm{g}, 14 \sim 1 \mathrm{~d})\end{array}$ & $\begin{array}{c}\text { Body weight } \\
(\mathrm{g}, 21 \mathrm{~d})\end{array}$ & $\begin{array}{c}\text { Body weight gain } \\
(\mathrm{g}, 21 \sim 14 \mathrm{~d})\end{array}$ \\
\hline Control & $22.7 \pm 0.4$ & $3.2 \pm 0.7$ & $24.9 \pm 0.4$ & $2.2 \pm 0.3$ \\
Fe-def & $21.4 \pm 0.2$ & $1.8 \pm 0.5^{*}$ & $22.8 \pm 0.4$ & $1.4 \pm 0.3^{*}$ \\
FAS & $21.3 \pm 0.2$ & $1.7 \pm 0.4^{*}$ & $22.5 \pm 0.5$ & $1.2 \pm 0.8$ \\
APO & $21.0 \pm 0.1$ & $1.9 \pm 0.2^{*}$ & $22.6 \pm 0.3$ & $1.6 \pm 0.4$ \\
FER & $21.7 \pm 0.3$ & $1.7 \pm 0.7^{*}$ & $23.7 \pm 0.6$ & $2.0 \pm 0.2^{\dagger}$ \\
\hline
\end{tabular}

Fe-def: AIN Fe-deficient diet (3 mg Fe/kg diet) for 21 day (d), FAS: AIN Fe-deficient diets plus ferrous ammonium sulfate (100 mg/kg, p.o., once in $14 \mathrm{~d}$ ), APO: AIN Fe-deficient diet plus ferritin-producing recombinant yeast ( $3 \mathrm{~g} / \mathrm{kg}$, p.o., once in $14 \mathrm{~d})$, FER: AIN Fe-deficient diets plus APO reacted with iron $\left(\mathrm{Fe}^{2+}\right)(3 \mathrm{~g} / \mathrm{kg}$, p.o., once in $14 \mathrm{~d})$. Tukey's test showed significant differences among group in body weight gain. Each value is the mean \pm SEM of 7 animals. ${ }^{*} p<0.05 v$ s. control, ${ }^{\dagger} p<0.05 v s$. Fe-def.

Table 2. Changes in hemoglobin value and hematocrit ratio

\begin{tabular}{cccccc}
\hline \hline & Control & Fe-def & FAS & APO & FER \\
\hline $\mathrm{Hb}(\mathrm{g} / \mathrm{dL})$ & $13.1 \pm 0.2$ & $9.8 \pm 0.5^{*}$ & $12.1 \pm 0.6^{\dagger}$ & $14.2 \pm 0.3^{\dagger}$ & $14.5 \pm 0.5^{\dagger}$ \\
$\mathrm{Ht}(\%)$ & $39.5 \pm 0.8$ & $32.9 \pm 1.1^{*}$ & $33.8 \pm 1.7$ & $42.2 \pm 1.1^{\dagger}$ & $40.8 \pm 0.6^{\dagger}$ \\
\hline
\end{tabular}

Fe-def: AIN Fe-deficient diet (3 mg Fe/kg diet) for $21 \mathrm{~d}$, FAS: AIN Fe-deficient diets plus ferrous ammonium sulfate (100 mg/kg, p.o., once in $14 \mathrm{~d}$ ), APO: AIN Fe-deficient diet plus ferritin-producing recombinant yeast (3 g/kg, p.o., once in $14 \mathrm{~d})$, FER: AIN Fe-deficient diets plus APO reacted with iron $(3 \mathrm{~g} / \mathrm{kg}$, p.o., once in $14 \mathrm{~d})$. Each value is the mean \pm SEM of 7 animals. Tukey's test showed significant differences among group in level of hemoglobin and hematocrit. ${ }^{*} p<0.05 v s$. control, ${ }^{\dagger} p<0.05$ vs. Fe-def. 
A

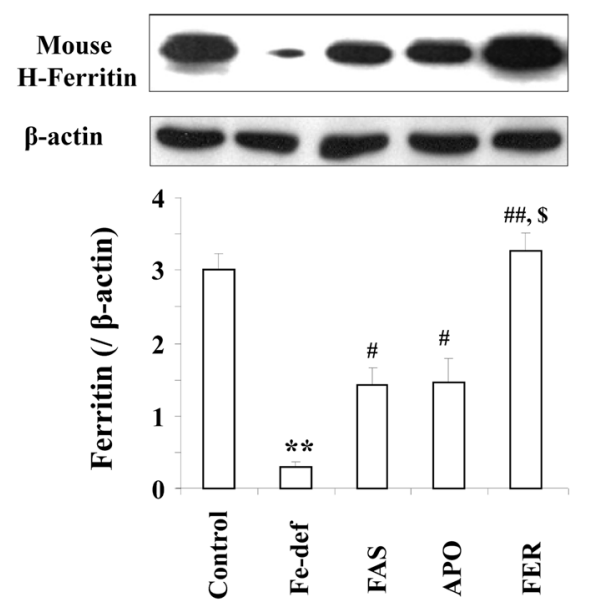

B

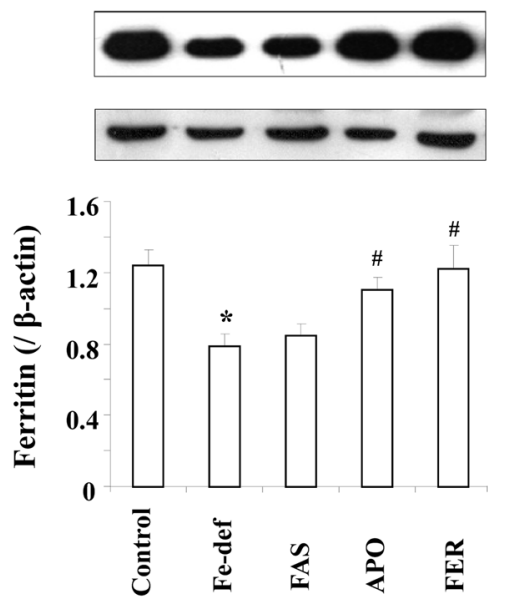

Fig. 2. Changes in ferritin heavy-chain expression in mice spleen (A) and liver (B). Fe-def, AIN Fe-deficient diet (3 mg Fe/kg diet) for $21 \mathrm{~d}$; FAS, AIN Fe-deficient diets plus ferrous ammonium sulfate (100 mg/kg, p.o., once in $14 \mathrm{~d})$; APO, AIN Fe-deficient diet plus ferritin-producing recombinant yeast (3 g/kg, p.o., once in $14 \mathrm{~d})$; FER, AIN Fe-deficient diets plus APO reacted with iron (3 g/ $\mathrm{kg}$, p.o., once in $14 \mathrm{~d})$. Proteins $(30 \mu \mathrm{g} / \mathrm{lane})$ were used for sodium dodecyl sulfate-polyacrylamide gel electrophoresis. Each value is the mean \pm SEM of 7 animals. Tukey's test showed significant differences among group in H-ferritin expression. ${ }^{*} p<0.05 v s$. control, ${ }^{* *} p<0.01$ vs. Control, ${ }^{\#} p<0.05$ vs. Fe-def, ${ }^{\#} p<0.01$ vs. Fe-def. ${ }^{\$} p<0.05$ vs. APO.

Table 3. Tissue iron stores in iron deficiency mice

\begin{tabular}{|c|c|c|}
\hline & \multicolumn{2}{|c|}{ Iron concentration in tissues $(\mathrm{mmol} / \mathrm{g})$} \\
\hline & Spleen & Liver \\
\hline Control & $3.73 \pm 0.32$ & $1.75 \pm 0.10$ \\
\hline Fe-def & $1.80 \pm 0.10^{*}$ & $1.10 \pm 0.19^{*}$ \\
\hline FAS & $2.54 \pm 0.18^{\dagger}$ & $1.26 \pm 0.13$ \\
\hline APO & $2.60 \pm 0.11^{\dagger}$ & $1.57 \pm 0.15^{\dagger}$ \\
\hline FER & $3.41 \pm 0.22^{\dagger, \hbar \S}$ & $1.70 \pm 0.14^{\dagger}$ \\
\hline
\end{tabular}

Fe-def: AIN Fe-deficient diet (3 mg Fe/kg diet) for $21 \mathrm{~d}$, FAS: AIN Fe-deficient diets plus ferrous ammonium sulfate $(100 \mathrm{mg} / \mathrm{kg}$, p.o., once in $14 \mathrm{~d}$ ), APO: AIN Fe-deficient diet plus ferritin-producing recombinant yeast $(3 \mathrm{~g} / \mathrm{kg}$, p.o., once in $14 \mathrm{~d})$, FER: AIN Fe-deficient diets plus APO reacted with iron $(3 \mathrm{~g} / \mathrm{kg}$, p.o., once in $14 \mathrm{~d})$. Each value is the mean \pm SEM of 7 animals. Tukey's test showed significant differences among group in iron stores. ${ }^{*} p<$ $0.05 v s$. control, ${ }^{\dagger} p<0.05 v$ s. Fe-def, ${ }^{\star} p<0.05 v s$. FAS, ${ }^{\S} p<0.05$ vs. APO.

istered APO showed a significant enhancement in ferritin heavy-chain levels both in the spleen and in the liver $(p<$ 0.05 vs. Fe-def). Ferritin heavy-chain levels in FER treated mice was significantly higher than in APO treated mice in the spleen $(p<0.01$ vs. Fe-def: $p<0.05$ vs. APO) but not in the liver. The western blot analysis results from the ferritin heavy-chain were consistent with the results from iron concentration in spleen and liver.

\section{Discussion}

Our major finding is that treatment of anemic mice with iron accumulated yeast cells producing Sus scrofa ferritin heavy-chain (FER) significantly improves iron deficiency by enhancing the levels of $\mathrm{H}$-ferritin in the spleen and liver. Consistent with this result we found that FER treatment restored iron concentration in these tissues. In addition, FER treatment significantly increased hemoglobin value and hematocrit ratio and enhanced body weight gain. Thus, our results suggest that treatment with iron accumulated yeast cells producing Sus scrofa H-ferritin will be an important strategy to recover anemic piglets.

Ferritin can interact readily with and aggregate Fe (II) ions in solution under aerobic conditions [10]. Seo et al. [17] reported that the construction of recombinant $S$. cerevisiae produces human ferritin heavy-chain. They reported that the recombinant $S$. cerevisiae expressing human ferritin heavychain genes accumulated $16.7 \mu \mathrm{mol} / \mathrm{g}$ of iron per wet cell weight after reaction with $14.3 \mathrm{mM}$ of Fe [16]. In the current study, we developed recombinant $S$. cerevisiae producing $S u s$ scrofa ferritin heavy-chain. These recombinant yeast cells also reacted with $14.3 \mathrm{mM}$ of $\mathrm{Fe}\left(\mathrm{NH}_{4}\right)_{2}\left(\mathrm{SO}_{4}\right)_{2}$.

Although the effective mechanism of ferritin on iron deficiency animal models remains to be further elucidated, this bioavailability may be, at least in part, related to the iron aggregation in ferritin. In a study, Chang et al. [3] demonstrated that ferritin reacted with iron was effective in inducing recovery in anemic rats. We observed that body weight gain was significantly decreased in Fe-deficient groups and that treatment with FER improved body weight gain. However, FAS or APO did not recover body weight gain. In a report using iron deficient mice, Change et al. [3] demonstrated that the rat body weight increased when orally admin- 
istered with human ferritin expressing yeast and iron. However, the body weight did not significantly increase when given ferritin expressing yeast or iron alone. Similarly, anemic rats gained body weight only when given both transgenic rice expressing soybean ferritin and iron [1]. These results suggest that only the combination of ferritin and iron can enhance body weight gain. Our data are in agreement with these observations. To our knowledge, this is the first study to demonstrate that iron accumulated yeast cells producing Sus scrofa H-ferritin significantly recovers body weight gain.

In the current study, no significant difference was observed in hemoglobin value and hematocrit ratio between APO and FER treatment, although each administration did significantly restores these values. Moreover, the APO treated group showed elevated levels of hemoglobin and hematocrit comparable to the control mice group although no iron was added. It is unclear what mechanisms are involved in this process. We tentatively suggest the possibility that uptake of APO in the mouse gastrointestinal tract preferentially promoted H-ferritin synthesis in these two tissues. In the FAS group, hemoglobin value was similar to that in the control group, whereas the hematocrit ratio was not recovered. The reason for decreased hematocrit ratio in FAS is required further detailed studies.

The bioavailability of recombinant yeast cells producing Sus scrofa ferritin heavy-chain on iron storage in tissues was determined by measuring iron concentration and mouse ferritin levels. We observed that treatment with FAS, APO or FER considerably enhanced the iron concentration in spleen. These results are consistent with ferritin expression in the spleen. In addition, there was a significantly higher increase in the iron concentration and ferritin protein levels in the spleen of FER group compared with that in FAS or APO group. The results suggest that the treatment with FER might potentiate iron accumulation followed by more increased ferritin formation. Interestingly, in our experimental system, FAS treatment did not significantly enhance iron concentration in the liver compared with that in FER or APO group. The present result is consistent with earlier findings [3] showing that FAS did not sufficiently restore iron concentration in rat livers. Furthermore, the change in iron concentration was consistent with ferritin levels in the liver. The underlying positive effects of FAS and APO in our experimental system remain to be fully determined but the current results indicate that treatment with FER would be a major source of bioavailable iron through enhanced ferritin formation in the spleen.

It is well known that the human ferritin heavy-chain expressed in the recombinant yeast is a major iron-binding protein $[17,18]$. The iron and ferritin composition of soybean seeds improved iron concentration in iron deficient rats [1]. Iron in transgenic rice expressing ferritin has exerted a positive effect on iron concentrations in spleen and liver [15]. However, the exact mechanism of iron absorption is still unclear. We observed here that the treatment with FER sig- nificantly recovered iron concentration and increased ferritin protein levels in the spleen and liver. Therefore, we raise the possibility that iron concentration induced by FER may affect ferritin formation and that this formation might contribute to the enhanced storage of iron in spleen and liver, consequently leading to body weight restoration.

In conclusion, our results suggest that the iron accumulated in the recombinant ferritin of yeast can contribute as a source of iron and can be used in feed supplement for anemic piglets.

\section{Acknowledgments}

This research was financially supported by the ministry of knowledge economy (MKE) and Korea institute for advancement of technology (KIAT) through the research and development for regional industry.

\section{References}

1. Beard JL, Burton JW, Theil EC. Purified ferritin and soybean meal can be sources of iron for treating iron deficiency in rats. J Nutr 1996, 126, 154-160.

2. Brady PS, Ku PK, Ullrey DE, Miller ER. Evaluation of an amino acid-iron chelate hematinic for the baby pig. J Anim Sci 1978, 47, 1135-1140.

3. Chang YJ, Jo MY, Hwang EH, Park CU, Kim KS. Recovery from iron deficiency in rats by the intake of recombinant yeast producing human $\mathrm{H}$-ferritin. Nutrition 2005, 21, 520-524.

4. Clark SF. Iron deficiency anemia: diagnosis and management. Curr Opin Gastroenterol 2009, 25, 122-128.

5. Collard KJ. Iron homeostasis in the neonate. Pediatrics 2009, 123, 1208-1216.

6. Egeli AK, Framstad T. An evaluation of iron-dextran supplementation in piglets administered by injection on the first, third or fourth day after birth. Res Vet Sci 1999, 66, 179-184.

7. Furugouri K, Miyata Y, Shijimaya K, Narasaki N. Developmental changes in serum ferritin of piglets. J Anim Sci 1983, 57, 960-965.

8. Gasche C, Lomer MC, Cavill I, Weiss G. Iron, anaemia, and inflammatory bowel diseases. Gut 2004, 53, 1190-1197.

9. Gietz GP, Woods RA. Transformation of yeast by lithium acetate/single-stranded carrier DNA/polyethylene glycol method. Methods Enzymol 2002, 350, 87-96.

10. Harrison PM, Arosio P. The ferritins: molecular properties, iron storage function and cellular regulation. Biochim Biophys Acta 1996, 1275, 161-203.

11. Jung BD, Shin EJ, Nguyen XK, Jin CH, Bach JH, Park SJ, Nah SY, Wie MB, Bing G, Kim HC. Potentiation of methamphetamine neurotoxicity by intrastriatal lipopolysaccharide administration. Neurochem Int 2010, 56, 229-244.

12. Linder MC, Moor JR, Scott LE, Munro HN. Prenatal and postnatal changes in the content and species of ferritin in rat liver. Biochem J 1972, 129, 455-462.

13. Lipiñski P, Starzyñski RR, Canonne-Hergaux F, Tudek 
B, Oliñski R, Kowalczyk P, Dziaman T, Thibaudeau O, Gralak MA, Smuda E, Woliñski J, Usiñska A, Zabielski R. Benefits and risks of iron supplementation in anemic neonatal pigs. Am J Pathol 2010, 177, 1233-1243.

14. May ME, Fish WW. The isolation and properties of porcine ferritin and apoferritin. Arch Biochem Biophys 1977, 182, 396-403.

15. Murray-Kolb LE, Takaiwa F, Goto F, Yoshihara T, Theil EC, Beard JL. Transgenic rice is a source of iron for iron-depleted rats. J Nutr 2002, 132, 957-960.

16. Seo HY, Chung YJ, Kim SJ, Park CU, Kim KS. Enhanced expression and functional characterization of the human ferritin $\mathrm{H}$ - and L-chain genes in Saccharomyces cerevisiae. Appl Microbiol Biotechnol 2003, 63, 57-63.

17. Seo HY, Jeon ES, Chung YJ, Kim KS. Heterologous expression of human ferritin H-chain and L-chain genes in Saccharomyces cerevisiae. KSBB Journal 2002, 17,162-168.

18. Svoboda M, Drabek J. Iron deficiency in suckling piglets: etiology, clinical aspects and diagnosis. Folia Vet 2005, 49, 104-111.

19. Theil EC. Ferritin: structure, gene regulation, and cellular function in animals, plants, and microorganisms. Annu Rev Biochem 1987, 56, 289-315. 